\title{
Lecithin-cholesterol acyltransferase (LCAT) activity is lower in patients with type 2 diabetes in the presence of metabolic syndrome
}

\section{Armin Rajab', Soghra Rabizadeh ${ }^{1}$, Masoume Bitaraf' ${ }^{1}$, Azam Ghanei ${ }^{2}$, Alireza Esteghamati $^{1}$, Manouchehr Nakhjavani ${ }^{1}$, Hossein Mrmiranpour ${ }^{1}$}

${ }^{1}$ Endocrinology and Metabolism Research Center (EMRC), Vali-Asr Hospital, Tehran University of Medical Sciences, Tehran, Iran; ${ }^{2}$ Diabetes Research Center, Shahid Sadoughi University of Medical Sciences, Yazd, Iran

*Corresponding Author: Hossein Mirmiranpour, M.D., PhD, Endocrinology and Metabolism Research Center (EMRC), Vali-Asr Hospital, School of Medicine, Tehran University of Medical Sciences, Tehran, Iran

Submission Date: January $30^{\text {th }}$, 2020; Acceptance Date: April $25^{\text {th }}$, 2020, Publication Date: April $30^{\text {th }}, 2020$

Citation: Rajab A., Rabizadeh S., Bitaraf M., Ghanei A., Esteghamati A., Manouchehr Nakhjavani M., Mrmiranpour H. Lecithin-cholesterol acyltransferase (LCAT) activity is lower in patients with type 2 diabetes in the presence of metabolic syndrome. Bioactive Compounds in Health and Disease 2020; 3(4): 66-73. DOI: https://doi.org/10.31989/bchd.v3i4.690

\begin{abstract}
Backgrounds: Metabolic syndrome can facilitate the development of diabetes mellitus and has gained a negative reputation for its adverse effects on the general health of the population and its tendency to increase the risk of cardiovascular diseases. Cardiovascular complications may be mediated by the alteration of certain enzyme activities and pathways. Lecithin-Cholesterol Acyltransferase (LCAT) may have a potential role in the prevention of atherosclerosis as a ratelimiting enzyme in reverse cholesterol transport. In this study, LCAT activity was compared among patients with type 2 diabetes in the presence or absence of metabolic syndrome.
\end{abstract}

Methods: In the present study, 55 patients with type 2 diabetes with metabolic syndrome (DM+MS group), 25 patients with type 2 diabetes without metabolic syndrome (DM-MS group), and a control group of 43 subjects without type 2 diabetes (HC group) were compared based on their anthropometric parameters, lipid profiles, glycemic indices, and LCAT activity.

Results: LCAT activity was lower among participants of the DM+MS group (70.22 \pm 7.88$)$ in comparison with the both HC group $(89.51 \pm 3.59)$ and the DM-MS group (76.73 \pm 7.25$)$ (respectively $\mathrm{P}<0.001, \mathrm{P}=0.021$ ). There was no significant difference in the fasting blood glucose levels, as evidenced by the HbA1c and HOMA-IR measurements between the $\mathrm{DM}+\mathrm{MS}$ group and the DM-MS group. BMI was significantly higher in the DM+MS group compared to the DM-MS group $(\mathrm{P}=0.031)$. In patients with type 2 diabetes mellitus and metabolic syndrome, there was a negative correlation between LCAT activity and BMI ( $\mathrm{r}=-$ $0.275, \mathrm{P}=0.048$ ). There was also a negative correlation between LCAT activity and HbA1c in the DM-MS group $(\mathrm{r}=-0.606, \mathrm{P}=0.008)$ and the $\mathrm{DM}+\mathrm{MS}$ group $(\mathrm{r}=-0.421, \mathrm{P}=0.002)$.

Conclusion: LCAT activity was lower among patients with type 2 diabetes compared to subjects without type 2 diabetes and it had a greater reduction in the presence of metabolic 
syndrome. LCAT activity also had a negative correlation with BMI in patients with type 2 diabetes mellitus and metabolic syndrome compared to patients with type 2 diabetes that did not have metabolic syndrome.

Keywords: Lecithin-Cholesterol Acyltransferase (LCAT), Metabolic syndrome, Diabetes mellitus, type 2, BMI

\section{INTRODUCTION:}

Metabolic syndrome is a serious condition defined by the concurrence of obesity, dyslipidemia, insulin resistance, and elevated blood pressure [1]. It directly amplifies the risk of cardiovascular accidents, atherosclerotic vascular diseases, and type 2 diabetes mellitus [2]. In addition, the prevalence of metabolic syndrome is increasing rapidly worldwide, especially among developing countries such as those in the Middle East [3]. Therefore metabolic syndrome imposes a great burden on the general health of populations and has negative socioeconomic impacts [4]. Numerous researchers have illustrated that changes in lifestyle, specifically diet, can predispose people to metabolic syndrome [5].

One of the components of metabolic syndrome is dyslipidemia which is characterized by high levels of serum triglyceride (TG) and low levels of serum high-density lipoprotein cholesterol (HDL-C) [6]. Researchers have shown that the lecithin cholesterol acyltransferase (LCAT) enzyme helps to produce HDL by transferring 2-acyl groups from lecithin or phosphatidylethanolamine to unesterified cholesterol (UC) [7]. According to these researchers, LCAT is believed to play a major role in macrophage reverse cholesterol transport (RCT). Hence, alteration in LCAT activity can contribute significantly to atherosclerotic cardiovascular diseases [8].

The purpose of this study was to determine the independent correlates of LCAT activity in patients with both type 2 diabetes and metabolic syndrome in comparison with type 2 diabetic patients without metabolic syndrome and healthy controls. Though the LCAT activity in patients with type 2 diabetes mellitus was previously studied, there is a lack of information about diabetic patients with metabolic syndrome [9]. Understanding the implications of this condition can facilitate a better understanding of the pathophysiology of metabolic syndrome for clinicians and researchers.

\section{METHODS:}

A case-control study was performed in the Diabetes Clinic of Imam Khomeini Hospital (Tehran, Iran). In total, 80 patients (46 females and 34 males) with type 2 diabetes who came to our diabetes clinic for routine follow up were enrolled in the study. Additionally, a total of 50 adult volunteers with established levels of FBS $<126 \mathrm{mg} / \mathrm{dl}$ and without a history of diabetes mellitus were recruited. These volunteers were matched for gender, age, and body mass index (BMI) with the previously selected patients. After measurements were taken, all of the participants were divided into three different groups based on if they had diabetes and metabolic syndrome according to NCEP ATP III criteria (The US National Cholesterol Education Programme Adult Treatment Panel III). The patients needed to match any three of the five following criteria: 1 . Waist circumference $>102 \mathrm{~cm}$ for males and $>88 \mathrm{~cm}$ for females; 2. Fasting glucose $>100 \mathrm{mg} / \mathrm{dl} ; 3$. TG $>150 \mathrm{mg} / \mathrm{dl} ; 4$. HDL cholesterol $<40 \mathrm{mg} / \mathrm{dl}$ for males and $<50 \mathrm{mg} / \mathrm{dl}$ for females; 5 . Systolic blood pressure $>130 \mathrm{mmHg}$ or diastolic blood pressure $>85 \mathrm{mmHg}$ [6]. Seven participants without diabetes had been eliminated from the study due to metabolic syndrome. Therefore, a total of 69 females and 54 males were aligned into three groups: 55 participants with both metabolic syndrome and type 2 diabetes mellitus (DM+MS group); 25 participants with type 2 diabetes but without metabolic syndrome (DM-MS group); 
and 43 subjects without type 2 diabetes and metabolic syndrome as the control group (HC group).

Exclusion Criteria: Smoking, a positive history of cardiovascular, thyroid, or liver disease, pregnancy, an account of malignancy and proteinuria or renal involvement (creatinine $>1.5$ $\mathrm{mg} / \mathrm{dl}$ or glomerular filtration rate $<70 \mathrm{ml} / \mathrm{min}$ ).

The following variables were determined for each participant: age, gender, diabetes duration, body mass index (BMI; $\mathrm{kg} / \mathrm{m} 2$ ) according to the Quetelet equation, waist-to-hip ratio (WHR; waist circumference divided by hip circumference), blood pressure (systolic and diastolic), fasting blood sugar (FBS), HbA1c, total cholesterol (TC), LDL-C, HDL-C, triglycerides (TG), plasma creatinine $(\mathrm{Cr})$, fasting plasma insulin (Ins), homeostasis model assessment of insulin resistance (HOMA-IR) [10], medications, and LCAT activity.

Blood pressure was documented using an electronic device on the right arm in the sitting position after 5 minutes of rest. Systolic (SBP) and diastolic (DBP) blood pressures were calculated from two recordings with a minimal interval of 10 minutes.

The following blood sample measurements were conducted based on the routine processes of our clinic [11]. Venous blood was taken at 08:00 h after the subjects were fasting for 12 hours. The blood was obtained by direct puncture to the antecubital vein while the subjects were in supine rest for 30 minutes. They were urged not to change dietary habits in the week preceding the study. Venous blood was collected into ethylene diamine tetracetic acidcontaining $(1.5 \mathrm{mg} / \mathrm{ml})$ tubes and placed on ice immediately. Plasma was obtained within 30 minutes by centrifugation at $3000 \mathrm{rpm}$ for $15 \mathrm{~min}$ at $4^{\circ} \mathrm{C}$. Total cholesterol, LDL-C, HDL-C, and TG levels were then measured by direct enzymatic colorimetry using a Technicon RAanalyzer (Pars Azmoon, Karaj, Iran). HbA1c level was determined by high-performance liquid chromatography (HPLC) and plasma insulin was measured using an immunoradiometric assay kit (Immunotec IRMA, Czech Republic; intra- and inter-assay precision: $\mathrm{CV}=<4.3$ and $=<3.4 \%$, respectively). To approximate insulin resistance (IR), the Homeostasis Model Assessment (HOMA) index was calculated as follows: HOMA-IR= FBS $(\mathrm{mg} / \mathrm{dl}) \times$ insulin $(\mathrm{mu} / \mathrm{L}) / 405$. Glomerular filtration rate (GFR) was estimated using the Cockcroft-gault formula. The plasma LCAT activity level was measured using a commercially available kit (Roar Biomedical Inc.). Plasma was incubated with a fluorescent substrate and the fluorescence intensity of the intact substrate was measured at $470 \mathrm{~nm}$ by a Hitachi F-4500 Fluorescence Spectrophotometer. As the substrate is hydrolyzed by LCAT, a monomer is produced which becomes detectable at $390 \mathrm{~nm}$. LCAT activity was calculated by measuring the change in $470 / 390 \mathrm{~nm}$ emission intensity.

Ethical approval: All participants gave their written informed consent and the local ethics committee at Tehran University of Medical Sciences preapproved the study protocol. Additionally, all procedures were in accordance with the 1964 Helsinki declaration and its later amendments or comparable ethical standards.

Statistical analysis: Statistical package for the social science program (SPSS for Windows Inc. version 22. Chicago, Illinois, USA) was used to perform statistical analyses. Continuous variables were expressed as mean \pm standard deviation (SD). As quantitative variables had a normal distribution, Student's t-test and one-way ANOVA and MANOVA analysis were conducted to compare mean levels of variables between groups. Chi-squared test was applied to compare categorical variables and the Mann-Whitney $U$ test was used to assess the differences of non-parametric variables such as duration of diabetes. The associations between LCAT activity and independent variables were examined using Pearson's correlation test. Two-sided P-values $<0.05$ were considered statistically significant. 


\section{RESULTS:}

The baseline characteristics of the study population are presented in Table 1 .

Table 1. Demographic, clinical, and laboratory data of different groups

\begin{tabular}{|c|c|c|c|c|}
\hline & $\begin{array}{l}\text { Patients without } \\
\text { type } 2 \text { diabetes } \\
\text { and metabolic } \\
\text { syndrome }(N=43)\end{array}$ & $\begin{array}{l}\text { Patients with type } \\
2 \text { diabetes without } \\
\text { metabolic } \\
\text { syndrome }(\mathrm{N}=25)\end{array}$ & $\begin{array}{l}\text { Patients with type } 2 \\
\text { diabetes and } \\
\text { metabolic syndrome } \\
(\mathrm{N}=55)\end{array}$ & P. Value \\
\hline Female/Male n $(\%)$ & $23(53.5) / 20(46.5)$ & $12(48.0) / 13(52.0)$ & $34(61.8) / 21(38.2)$ & 0.154 \\
\hline Age, mean \pm SD years & $49.98 \pm 6.78$ & $47.71 \pm 6.60$ & $50.87 \pm 6.78$ & 0.192 \\
\hline $\mathrm{BMI}$, mean $\pm \mathrm{SD} \mathrm{kg} / \mathrm{m}^{2}$ & $27.11 \pm 2.90$ & $25.72 \pm 2.35 *$ & $27.95 \pm 3.76 \sqrt{ }$ & 0.029 \\
\hline Waist, mean \pm SD cm & $92.85 \pm 9.21 *$ & $90.70 \pm 7.83 *$ & $99.04 \pm 8.83 \# \sqrt{ }$ & $<0.001$ \\
\hline WHR, mean \pm SD & $0.92 \pm 0.07 *$ & $0.95 \pm 0.05$ & $0.99 \pm 0.05 \#$ & $<0.001$ \\
\hline $\mathrm{SBP}$, mean $\pm \mathrm{SD} \mathrm{mmHg}$ & $123.58 \pm 10.90$ & $117.62 \pm 12.61 *$ & $126.25 \pm 13.22 \sqrt{ }$ & 0.026 \\
\hline $\mathrm{DBP}$, mean $\pm \mathrm{SD} \mathrm{mmHg}$ & $77.28 \pm 8.47$ & $72.38 \pm 7.68 *$ & $78.39 \pm 8.95 \sqrt{ }$ & 0.025 \\
\hline $\begin{array}{l}\text { Duration of DM, median } \\
(\mathrm{Q} 25 \%-75 \%) \text { Years }\end{array}$ & - & $3.0(2.0-6.5)$ & $4.0(2.0-5.7)$ & 0.385 \\
\hline $\mathrm{FBS}$, mean $\pm \mathrm{SD} \mathrm{mg} / \mathrm{dl}$ & $87.56 \pm 7.51 * \sqrt{ }$ & $195.76 \pm 66.89 \#$ & $192.62 \pm 67.34 \#$ & $<0.001$ \\
\hline $\mathrm{HbA} 1 \mathrm{c}$, mean $\pm \mathrm{SD} \%$ & $4.90 \pm 0.43 * \sqrt{ }$ & $8.61 \pm 2.24 \#$ & $9.05 \pm 2.20 \#$ & $<0.001$ \\
\hline $\mathrm{TG}, \mathrm{mean} \pm \mathrm{SD} \mathrm{mg} / \mathrm{dl}$ & $98.46 \pm 30.55^{*}$ & $128.81 \pm 48.08^{*}$ & $191.70 \pm 71.69 \# \sqrt{ }$ & $<0.001$ \\
\hline $\begin{array}{l}\text { Total Cholesterol, } \\
\text { mean } \pm \text { SD mg/dl }\end{array}$ & $201.91 \pm 22.14$ & $183.62 \pm 34.55$ & $200.77 \pm 46.09$ & 0.144 \\
\hline HDL-C, mean \pm SD mg/dl & $53.23 \pm 10.72 * \sqrt{ }$ & $46.28 \pm 8.53 \# *$ & $39.69 \pm 7.78 \# \sqrt{ }$ & $<0.001$ \\
\hline LDL-C, mean \pm SD mg/dl & $113.37 \pm 11.62$ & $119.05 \pm 18.71$ & $119.37 \pm 28.99$ & 0.386 \\
\hline $\mathrm{eGFR}$, mean $\pm \mathrm{SD} \mathrm{ml} / \mathrm{min}$ & $90.00 \pm 18.70$ & $99.89 \pm 11.71$ & $95.83 \pm 20.63$ & 0.275 \\
\hline $\begin{array}{l}\text { LCAT activity, mean } \pm \text { SD } \\
\mu \mathrm{mol} / \mathrm{L} / \mathrm{hr}\end{array}$ & $89.51 \pm 3.59 * \sqrt{ }$ & $76.73 \pm 7.25 \# *$ & $70.22 \pm 7.88 \# \sqrt{ }$ & $<0.001$ \\
\hline HOMA-IR, mean \pm SD & $1.32 \pm 0.83 * \sqrt{ }$ & $2.96 \pm 2.33 \#$ & $2.92 \pm 2.19 \#$ & $<0.001$ \\
\hline \multicolumn{5}{|l|}{ Medication \% (n) } \\
\hline Oral agents & - & $80 \%(20)$ & $78.2 \%(43)$ & \multirow{3}{*}{0.817} \\
\hline Insulin + Metformin & - & $12 \%(3)$ & $16.4 \%(9)$ & \\
\hline Insulin & - & $8 \%(2)$ & $5.5 \%(3)$ & \\
\hline Statin use & - & $96 \%(24)$ & $98.2 \%(54)$ & 0.594 \\
\hline Anti-Hypertensiv & - & $20 \%(5)$ & $30.9 \%(17)$ & 0.311 \\
\hline
\end{tabular}

BMI, Body mass index; WHR, Waist to Hip ratio; SBP, Systolic blood pressure; DBP, Diastolic blood pressure; DM, Diabetes mellitus; FBS, Fasting blood glucose; HbA1c, Hemoglobin A1C; TG, Triglyceride; HDLC, High-density lipoprotein cholesterol; LDL-C, Low-density lipoprotein cholesterol; eGFR, Estimated glomerular filtration rate; LCAT, Lecithin cholesterol acyltransferase; HOMA-IR, Homeostatic Model Assessment of Insulin Resistance index; n, Number.

* Significant difference with (DM positive, MS positive);

$\sqrt{ }$ Significant difference with (DM positive, MS negative);

\# Significant difference with (DM negative, MS negative) control. 
No significant differences were found among all groups regarding age, gender, total cholesterol, LDL-C, or eGFR. However, post-hoc analysis showed that, among patients with type 2 diabetes, the mean of all components of metabolic syndrome including SBP, DBP, waist, and TG were significantly higher in participants with metabolic syndrome compared to participants without it $(\mathrm{P}=0.027, \mathrm{P}=0.026, \mathrm{P}=0.002$, and $\mathrm{P}<0.001$, respectively $)$. On the other hand, the levels of HDL-C and LCAT activity were lower among participants of the DM+MS group in comparison with both the $\mathrm{HC}$ group and the DM-MS group (respectively $\mathrm{P}<0.001$, $\mathrm{P}=0.021$ for HDL-C and $\mathrm{p}<0.001, \mathrm{P}=0.031$ for LCAT activity). Although FBS, HbA1c, and HOMA-IR levels were higher among patients with type 2 diabetes compared with subjects without type 2 diabetes $(\mathrm{P}<0.001$ for all of them), there wasn't any significant difference between the $\mathrm{DM}+\mathrm{MS}$ and the DM-MS groups $(\mathrm{P}=0.975, \mathrm{P}=0.639, \mathrm{P}=0.997$ respectively). Additionally, BMI was significantly higher in the DM+MS group compared to the DM-MS group $(\mathrm{P}=0.031)$. The difference in duration of diabetes mellitus was not significant between the groups $(\mathrm{Z}=-0.868, \mathrm{P}=0.385)$ (Table 1$)$.

There were significant negative correlations between LCAT activity and HbA1c levels among both diabetic groups $(\mathrm{r}=-0.606, \mathrm{P}=0.008$ for the DM-MS group and $\mathrm{r}=-0.421, \mathrm{P}=0.002$ for the DM+MS group). Moreover, a significant negative correlation was found between LCAT activity and both the BMI and the amount triglycerides in $(\mathrm{r}=-0.275, \mathrm{P}=0.048)$. However, this correlation was not significant among participants of the DM-MS group. LCAT activity was also negatively correlated with the triglyceride levels among women in the $\mathrm{DM}+\mathrm{MS}$ group $(\mathrm{r}=-0.463, \mathrm{P}=0.013)$, but further analysis showed that this correlation disappeared after controlling by weight $(\mathrm{r}=-0.339, \mathrm{P}=0.236)$.

As shown, the level of LCAT activity was decreased by type 2 diabetes mellitus and metabolic syndrome $(\mathrm{F}=88.22, \mathrm{P}<0.001)$ (Figure 1). Further analysis showed that, although the mean level of LCAT activity among patients with type 2 diabetes declined steadily with the addition of more components of metabolic syndrome, it was not statistically significant $(\mathrm{F}=1.64, \mathrm{P}=0.174)$ (Figure 1).

Figure 1. The mean level of LCAT activity based on the components of metabolic syndrome

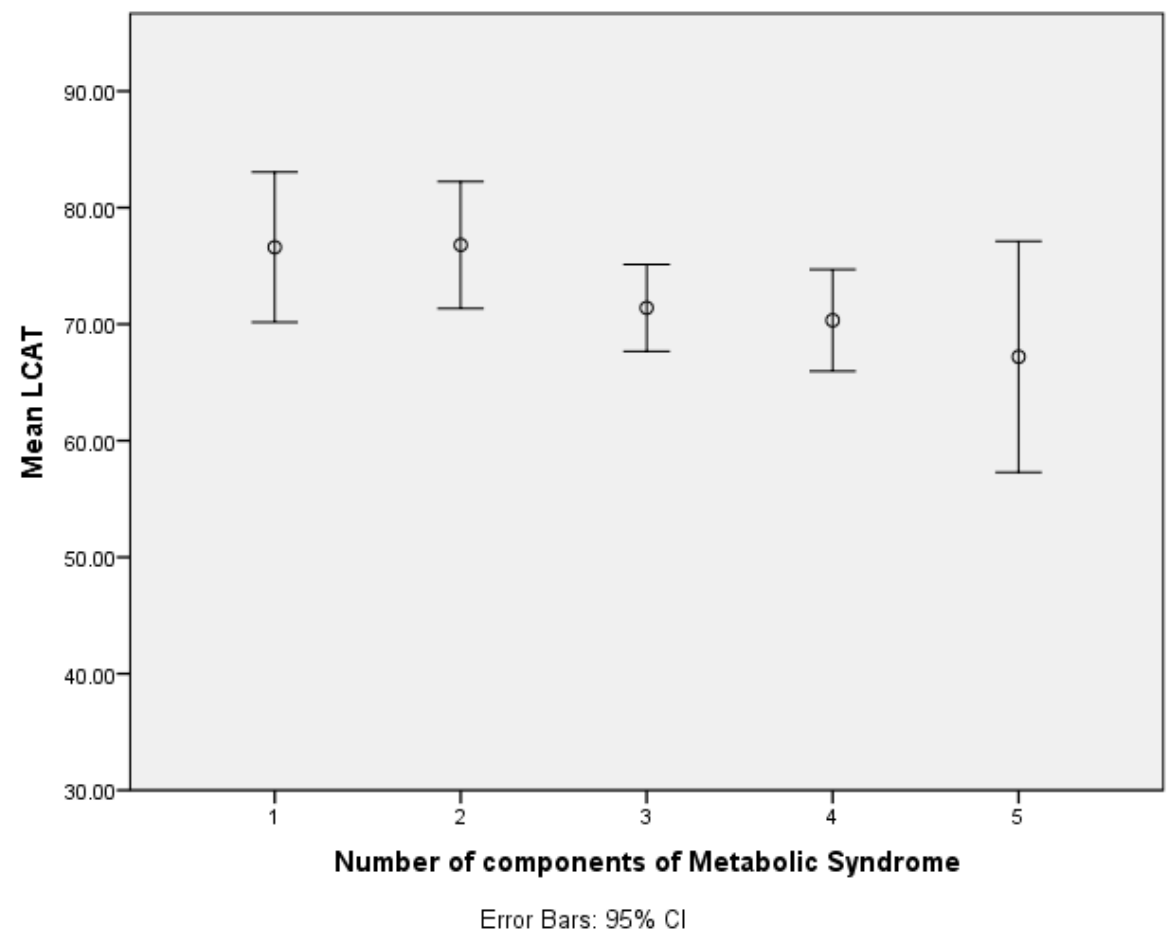

Mean LCAT, the mean of levels of LCAT enzyme activity; DM, diabetes mellitus;

MS, metabolic syndrome. 


\section{DISCUSSION:}

The results of this study showed decreased LCAT activity among people with type 2 diabetes mellitus compared to the healthy controls. Furthermore, LCAT activity declined steadily in the presence of metabolic syndrome. An increase in HbA1c was correlated with a decrease in LCAT activity. Currently, there is not enough information concerning LCAT activity specifically among type 2 diabetic patients that also have metabolic syndrome. One positive aspect of our study is that it compares diabetic patients with and without metabolic syndrome, minimizing the impact of diabetes and hyperglycemia on the LCAT activity. This allowed us to independently observe the effects of metabolic syndrome components on LCAT activity.

In patients with type 2 diabetes mellitus and metabolic syndrome, there was a negative correlation between LCAT activity and BMI. This correlation was not present in the healthy controls. The pathophysiology of metabolic syndrome is connected with visceral adiposity, which is the outcome of high caloric intake and lack of physical activity [12]. Visceral adiposity contributes to insulin resistance and obesity which together can increase susceptibility to hypertension, type 2 diabetes mellitus, dyslipidemia, and cardiovascular diseases [13]. Additionally, a previous study conducted on overweight-obese non-diabetic participants showed that BMI is inversely correlated with LCAT activity [14].

LCAT activity and HDL-C had the lowest levels in patients with type 2 diabetes and metabolic syndrome. We found a negative correlation between LCAT activity and triglyceride levels in women which disappeared after controlling for weight. Metabolic dyslipidemia is one of the components of metabolic syndrome related to either high levels of triglyceride or low HDL-C [2]. Lower levels of LCAT activity have also been associated with lower levels of HDL-C [15]. Moreover, the results of Nobecourt et al. indicated that hyperglycemia results in the glycation of apolipoprotein A-I (apoA-I), which is available in large quantities in HDL and has an important role in the activation of LCAT. Hence, hyperglycemia can adversely affect reverse cholesterol transport in patients with type 2 diabetes [16]. Awadallah et al. also suggested that LCAT activity decreased in the presence of diabetes and is correlated with haptoglobin phenotypes [17].

In this study, the level of LCAT activity in patients of the DM+MS and DM-MS groups was lower than that of subjects without type 2 diabetes. These findings are consistent with previous studies such as Kiziltunc et al. which reported that LCAT activity was decreased among diabetic patients with insulin-dependent or non-insulin-dependent diabetes mellitus [18]. This is also consistent with our earlier reports, which showed that LCAT activity was decreased in patients with type 2 diabetes compared to subjects without type 2 diabetes and negatively correlated with $\mathrm{HbA1c}$ [11]. On the other hand, there are a few controversial reports of increased LCAT activity in patients with metabolic syndrome [19, 20].

In terms of the study's limitations, we should mention the small sample size and the lack of a control group that suffered exclusively from metabolic syndrome. Moreover, we did not measure the level of oxidative and anti-oxidative biomarkers, however, this is something that can be fulfilled by further research.

One question that arises from this study is whether the insufficient activity of the LCAT enzyme predisposes people to metabolic syndrome or if the presence of metabolic syndrome could reduce the activity of LCAT. Further research should be done to investigate the exact relationship between metabolic syndrome and LCAT activity. Besides glycemic status, BMI could also potentially be a component of metabolic syndrome that alters LCAT activity. 


\section{CONCLUSION:}

LCAT activity was lower among patients with type 2 diabetes and it decreased more in the presence of metabolic syndrome. LCAT activity also had a negative correlation with BMI in patients with type 2 diabetes mellitus and metabolic syndrome compared to patients with type 2 diabetes and without metabolic syndrome. Therefore, controlling BMI may be a way to improve LCAT activity in type 2 diabetes mellitus.

List of abbreviations: BMI, Body mass index; WHR, Waist to Hip ratio; SBP, Systolic blood pressure; DBP, Diastolic blood pressure; DM, Diabetes mellitus; FBS, Fasting blood glucose; HbA1c, Hemoglobin A1C; TG, Triglyceride; HDL-C cholesterol, High-density lipoprotein; LDL-C, Low-density lipoprotein cholesterol; eGFR, Estimated glomerular filtration rate; LCAT, Lecithin cholesterol acyltransferase; HOMA-IR, Homeostatic Model Assessment of Insulin Resistance index; DM+MS group, Group of patients with type 2 diabetes mellitus and metabolic syndrome; DM-MS group, Group of patients with type 2 diabetes mellitus without metabolic syndrome; HC group, Group of patients without type 2 diabetes and metabolic syndrome as control group; MS, Metabolic syndrome.

Acknowledgments: Authors would like to appreciate the support and constructive comments of methodology research development office, Imam Khomeini hospital complex, Tehran, Iran

\section{Compliance with Ethical Standards:}

Conflict of Interest: The authors declare that they have no conflict of interest.

Informed consent: Informed consent was obtained from all individual participants included in the study

\section{REFERENCES:}

1. Grundy Scott, M., et al., Definition of Metabolic Syndrome. Circulation, 2004. 109(3): p. 433-438.

2. Kassi, E., et al., Metabolic syndrome: definitions and controversies. BMC Medicine, 2011. 9(1): p. 48.

3. Ansarimoghaddam, A., et al., Prevalence of metabolic syndrome in Middle-East countries: Meta-analysis of cross-sectional studies. Diabetes Metab Syndr, 2018. 12(2): p. 195-201.

4. Sherling, D.H., P. Perumareddi, and C.H. Hennekens, Metabolic Syndrome. J Cardiovasc Pharmacol Ther, 2017. 22(4): p. 365-367.

5. Mendrick, D.L., et al., Metabolic Syndrome and Associated Diseases: From the Bench to the Clinic. Toxicol Sci, 2018. 162(1): p. 36-42.

6. Huang, P.L., A comprehensive definition for metabolic syndrome. Disease models \& mechanisms, 2009. 2(5-6): p. 231-237.

7. Fielding, C.J. and P.E. Fielding, Molecular physiology of reverse cholesterol transport. J Lipid Res, 1995. 36(2): p. 211-28.

8. Kunnen, S. and M. Van Eck, Lecithin:cholesterol acyltransferase: old friend or foe in atherosclerosis? J Lipid Res, 2012. 53(9): p. 1783-99.

9. Nakhjavani, M., et al., HbA1c negatively correlates with LCAT activity in type 2 diabetes. Diabetes Res Clin Pract, 2008. 81(1): p. 38-41. 
10. Matthews, D.R., et al., Homeostasis model assessment: insulin resistance and betacell function from fasting plasma glucose and insulin concentrations in man. Diabetologia, 1985. 28(7): p. 412-9.

11. Ghanei, A., et al., Lecithin cholesterol acyltransferase activity is decreased in type 2 diabetes mellitus. Acta Medica Iranica, 2007. 45.

12. Matsuzawa, Y., T. Funahashi, and T. Nakamura, The Concept of Metabolic Syndrome: Contribution of Visceral Fat Accumulation and Its Molecular Mechanism. Journal of Atherosclerosis and Thrombosis, 2011. advpub: p. 11070704011107070401.

13. Rochlani, Y., et al., Metabolic syndrome: pathophysiology, management, and modulation by natural compounds. Therapeutic advances in cardiovascular disease, 2017. 11(8): p. 215-225.

14. Bajnok, L., et al., Relationship of endogenous hyperleptinemia to serum paraoxonase 1, cholesteryl ester transfer protein, and lecithin cholesterol acyltransferase in obese individuals. Metabolism, 2007. 56(11): p. 1542-1549.

15. Ossoli, A., C. Pavanello, and L. Calabresi, High-Density Lipoprotein, Lecithin: Cholesterol Acyltransferase, and Atherosclerosis. Endocrinology and metabolism (Seoul, Korea), 2016. 31(2): p. 223-229.

16. Nobecourt, E., et al., The impact of glycation on apolipoprotein A-I structure and its ability to activate lecithin:cholesterol acyltransferase. Diabetologia, 2007. 50(3): p. 643-53.

17. Awadallah, S., et al., Plasma levels of Apolipoprotein A1 and Lecithin:Cholesterol Acyltransferase in type 2 diabetes mellitus: Correlations with haptoglobin phenotypes. Diabetes Metab Syndr, 2017. 11 Suppl 2: p. S543-s546.

18. Kiziltunc, A., et al., Reduced lecithin: cholesterol acyltransferase (LCAT) and Na+, $\mathrm{K}+$, ATPase activity in diabetic patients. Clin Biochem, 1997. 30(2): p. 177-82.

19. Dullaart, R.P., et al., Plasma lecithin: cholesterol acyltransferase activity is elevated in metabolic syndrome and is an independent marker of increased carotid artery intima media thickness. J Clin Endocrinol Metab, 2008. 93(12): p. 4860-6.

20. Gruppen, E.G., et al., A novel protein glycan biomarker and LCAT activity in metabolic syndrome. Eur J Clin Invest, 2015. 45(8): p. 850-9. 